Bull. Austral. Math. Soc.

$60 \mathrm{~s} 27,60 \mathrm{~s} 35$

Vol. 43 (1991) [439-450]

\title{
ON THE CONSTRUCTION PROBLEM FOR SINGLE-EXIT MARKOV CHAINS
}

\author{
P.K. POLlETT
}

\begin{abstract}
I shall consider the following problem: given a stable, conservative, single-exit $q$ matrix, $Q$, over an irreducible state-space $S$ and a $\mu$-subinvariant measure, $m$, for $Q$, determine all $Q$-processes for which $m$ is a $\mu$-invariant measure. I shall provide necessary and sufficient conditions for the existence and uniqueness of such a process.
\end{abstract}

\section{INTRODUCTION}

The problem of constructing a Markov chain from its $q$-matrix of transition rates can be traced back to the work of Doob [4] in the late nineteen-forties. Since then, the problem has been considered by a number of authors. The major work was carried out in the fifties and early sixties by Feller $([5,6])$, Chung $([1,2])$, Reuter $([16,17,18])$ and Williams $([24,25])$ (see also $[3,4,10,11,12$ and 20]). This work culminated in the solution, by Williams [25], of the classical construction problem formulated by Feller in [6]. The problem is as follows : given a stable, conservative $q$-matrix, $Q=\left(q_{i j}, i, j \in S\right)$, over a countable state-space $S$, construct all $Q$-processes, that is identify all standard, time-homogeneous, continuous-time Markov chains taking values in $S$, with transition rates $Q$. The Feller minimal process provides an example of one such process. But, it is the possibility that this process might explode by performing infinitely many jumps in a finite time that creates interest in the construction problem, for, as Doob [4] showed, certain simple rules for restarting the process after an explosion give rise to an infinity of $Q$-processes.

The Feller minimal process is the unique $Q$-process if and only if $Q$ is regular, that is the equations

$$
\sum_{j \in S} q_{i j} x_{j}=\xi x_{i}, \quad i \in S,
$$

have no bounded, non-trivial solution (equivalently, non-negative solution), $x$, for some (and then for all) $\xi>0$ ([16]). When this condition fails there are infinitely many

Received 14 June 1990

I should like to thank Gopal Nair and Bevan Thompson for helpful discussions on this work.

Copyright Clearance Centre, Inc. Serial-fee code: 0004-9729/91 \$A2.00+0.00. 
$Q$-processes, including infinitely many honest ones ([16]), and the dimension, $d$, of the space of bounded vectors, $x$, on $S$ satisfying (1) (a quantity which does not depend on $\xi$ ), determines the number of "escape routes to infinity" available to the process. Williams [25] was able to provide a construction of all $Q$-processes under the assumption that $d$ is finite, following on from the work of Reuter $([17,18])$ who considered the single-exit case, $d=1$.

If $d$ is not assumed to be finite, little is known and the problem of finding all $Q$-processes appears to be very difficult, and remains unsolved. However, recently the problem has re-emerged and now attention is focused on finding one $Q$-process which satisfies a prescribed set of conditions. For example, it is of interest to know whether or not there exists an honest $Q$-process and, then, whether or not it is the unique honest $Q$-process. This question was first considered by Kendall [7] (see also Kendall and Reuter [8]) who used elegant but simple arguments based on the Hille-Yosida theorem from functional analysis. The most recent work centres on the assumption that one is given an invariant measure for the $q$-matrix. The problem is then to construct a process with $m$ as its invariant measure. It has particular significance if $\sum m_{i}<\infty$, for then one is looking for a process, which of necessity is honest, whose stationary distribution has been specified in advance. In this paper I shall provide necessary and sufficient conditions for there to exist a single-exit process for which a given measure, $m$, is $\mu$-invariant. Thus, although I shall deal with only a restricted class of processes, the invariance condition shall be weakened to $\mu$-invariance. The important special case of when $\mu=0$ is subsumed by the present study, although it was considered earlier in some detail (see [15]).

I hope that this work will provide some insight into how one should proceed in the more general setting, where the assumption that $Q$ be a single-exit $Q$-matrix is relaxed. I shall begin by collecting together various results on continuous-time Markov chains.

\section{Preliminaries}

I shall refer to a set $P(\cdot)=\left(p_{i j}(\cdot), i, j \in S\right)$, of real-valued functions defined on $[0, \infty)$, where $S$ is a countable set, as a standard transition function if

$$
\begin{gathered}
p_{i j}(t) \geqslant 0, \quad i, j \in S, t \geqslant 0, \\
\sum_{j \in S} p_{i j}(t) \leqslant 1, \quad i \in S, t \geqslant 0, \\
p_{i j}(s+t)=\sum_{k \in S} p_{i k}(s) p_{k j}(t), \quad i, j \in S, s, t \geqslant 0, \\
p_{i j}(0)=\delta_{i j}=\lim _{t \downarrow 0} p_{i j}(t), \quad i, j \in S .
\end{gathered}
$$


I shall refer to $P$ as being honest if equality holds in (3) for all $i \in S$. Condition (5) guarantees that, for all $i, j \in S, p_{i j}$ is uniformly continuous, as well as guaranteeing the existence of right-hand derivatives

$$
q_{i j}=p_{i j}^{\prime}(0)=\lim _{t \downarrow 0} \frac{p_{i j}(t)-\delta_{i j}}{t}
$$

with the property that $\quad 0 \leqslant q_{i j}<\infty, \quad j \neq i, i, j \in S$,

and

$$
\sum_{j \neq i} q_{i j} \leqslant-q_{i i} \leqslant \infty, \quad i \in S,
$$

the set $Q=\left(q_{i j}, i, j \in S\right)$ being called a $q$-matrix.

Henceforth I shall suppose that $Q$ is specified and I shall assume that $Q$ is stable, that is

and conservative, that is

$$
q_{i}:=-q_{i i}<\infty, \quad i \in S,
$$

$$
\sum_{j \in S} q_{i j}=0, \quad i \in S .
$$

For simplicity, any standard transition function, $P$, that satisfies

$$
p_{i j}^{\prime}(0)=q_{i j}, \quad i, j \in S,
$$

will be called a $Q$-function. Under the conditions I have imposed, any $Q$-function, $P$, satisfies the backward differential equations,

$$
p_{i j}^{\prime}(t)=\sum_{k \in S} q_{i k} p_{k j}(t)
$$

for all $i, j \in S$ and $t \geqslant 0$. The so-called Feller construction provides for the existence of a minimal solution, $F(\cdot)=\left(f_{i j}(\cdot), i, j \in S\right)$, to these equations, minimal in the sense that $f_{i j}(t) \leqslant p_{i j}(t)$ for all $t>0$ and all $i, j \in S$, where $P(\cdot)=\left(p_{i j}(\cdot), i, j \in S\right)$ is any $Q$-function. $F$ is also a $Q$-function and it satisfies the forward differential equations,

$$
p_{i j}^{\prime}(t)=\sum_{k \in S} p_{i k}(t) q_{k j}
$$

for all $i, j \in S$ and $t \geqslant 0$.

\section{The construction problem}

As mentioned in the introduction, I shall restrict my attention to the case where $Q$ is a single-exit $q$-matrix and so, henceforth, I shall suppose that the space of bounded, 
non-trivial, non-negative solutions to (1) has dimension 1. Under this condition, Reuter [17] identified all transition functions with a specified conservative $q$-matrix; for the non-conservative case see [18] and [26]. The problem is to determine for which of these transition functions is a specified measure $\mu$-invariant. In particular, I shall suppose that $m=\left(m_{j}, j \in S\right)$ is a specified $\mu$-subinvariant measure for $Q$, that is a collection of strictly positive numbers which satisfy

$$
\sum_{i \in S} m_{i} q_{i j} \leqslant-\mu m_{j}, \quad j \in S .
$$

For simplicity, I shall suppose that $S$ is irreducible for the minimal process, and, hence, for any other $Q$-process. For a $\mu$-subinvariant measure to exist, one must have that $0 \leqslant \mu \leqslant \lambda_{F}$, where $\lambda_{F}$ is the decay parameter of $S$ for $F$, the minimal $Q$-function (see [23] and [14]). The main result of the paper establishes necessary and sufficient conditions for there to exist a unique $Q$-function, $P$, such that $m$ is $\mu$-invariant for $P$, that is

$$
\sum_{i \in S} m_{i} p_{i j}(t)=e^{-\mu t} m_{j}
$$

for all $j \in S$ and $t \geqslant 0$; note that $m$ is said to be $\mu$-subinvariant for $P$ if

$$
\sum_{i \in S} m_{i} p_{i j}(t) \leqslant e^{-\mu t} m_{j}
$$

for all $j \in S$ and $t \geqslant 0$.

It will be convenient to present my results using Laplace transforms. Let $P$ be an arbitrary standard transition function and define the resolvent, $\Psi(\cdot)=\left(\psi_{i j}(\cdot), i, j \in S\right)$, of $P$ by

$$
\psi_{i j}(\alpha)=\int_{0}^{\infty} e^{-\alpha t} p_{i j}(t) d t, \quad i, j \in S
$$

this integral converges for all $\alpha>-\lambda_{P}$, where $\lambda_{P}$ is the decay parameter of $S$ for $P$ (see [9]). Analogous to (2)-(5), $\Psi$ satisfies

$$
\begin{gathered}
\psi_{i j}(\alpha) \geqslant 0, \quad i, j \in S, \alpha>0 \\
\sum_{j \in S} \alpha \psi_{i j}(\alpha) \leqslant 1, \quad i \in S, \alpha>0
\end{gathered}
$$

(9) the "resolvent equation"

$$
\begin{gathered}
\psi_{i j}(\alpha)-\psi_{i j}(\beta)+(\alpha-\beta) \sum_{k \in S} \psi_{i k}(\alpha) \psi_{k j}(\beta)=0, \quad i, j \in S, \alpha, \beta>0, \\
\lim _{\alpha \rightarrow \infty} \alpha \psi_{i j}(\alpha)=\delta_{i j}, \quad i, j \in S,
\end{gathered}
$$


and, any $\Psi$ which satisfies (7)-(10) is the resolvent of a standard transition function; for an elegant proof of this characterisation see [17] (see also [19]). Thus, there is a oneto-one correspondence between resolvents and standard transition functions. Further, (8) is satisfied with equality for all $i \in S$ and $\alpha>0$ if and only if $P$ is honest, in which case the resolvent is said to be honest. The $q$-matrix of $P$ can be recovered from $\Psi$ using the following identity:

$$
q_{i j}=\lim _{\alpha \rightarrow \infty} \alpha\left(\alpha \psi_{i j}(\alpha)-\delta_{i j}\right) .
$$

And, a resolvent that satisfies (11) is called a $Q$-resolvent. Explicit analogues of the backward and the forward equations will not be needed here. It will suffice to note that there is a one-to-one correspondence between $Q$-resolvents and $Q$-functions and that the resolvent, $\Phi(\cdot)=\left(\phi_{i j}(\cdot), i, j \in S\right)$, of the minimal $Q$-function, $F$, has itself a minimal interpretation (see [16] and [17]); for this reason $\Phi$ is called the minimal $Q$-resolvent.

The following result summarises Reuter's $[17]$ construction:

THEOREM 1. If $Q$ is a stable, conservative, single-exit $q$-matrix and if $\Psi$ is the resolvent of an arbitrary $Q$-function, $P$, then either $\Psi=\Phi$, the minimal $Q$-resolvent, or otherwise $\Psi$ must be of the form

where

$$
\begin{gathered}
\psi_{i j}(\alpha)=\phi_{i j}(\alpha)+z_{i}(\alpha) y_{j}(\alpha), \quad i, j \in S, \alpha>0 \\
z_{i}(\alpha)=1-\sum_{j \in S} \alpha \phi_{i j}(\alpha), \quad i \in S, \alpha>0
\end{gathered}
$$

The quantity $y(\alpha)=\left(y_{j}(\alpha), j \in S\right)$ must be of the form

$$
y_{j}(\alpha)=\frac{\eta_{j}(\alpha)}{c+\sum_{k \in S} \alpha \eta_{k}(\alpha)}, \quad j \in S, \alpha>0,
$$

where $c \geqslant 0$ and $\eta(\alpha)=\left(\eta_{j}(\alpha), j \in S\right)$ is a non-negative vector that satisfies

$$
\sum_{k \in S} \eta_{k}(\alpha)<\infty, \quad \alpha>0
$$

and

$$
\eta_{j}(\alpha)-\eta_{j}(\beta)+(\alpha-\beta) \sum_{k \in S} \eta_{k}(\alpha) \phi_{k j}(\beta)=0, \quad j \in S, \alpha, \beta>0 .
$$

$\Psi$ is honest if and only if $c=0$.

REMARKS: The theorem states that the resolvents of all processes with $q$-matrix $Q$ must be of the form (12). Indeed, once $\eta$ is specified, a family of $Q$-processes (exactly 
one of which is honest) is obtained by varying $c$. Thus, the problem of determining those $Q$-processes which satisfy a specified criterion amounts to determining which choices of $\eta$ and $c$ are admissible.

Expression (12) specifies $\Psi(\alpha)$ for all $\alpha>0$. However, the expression is valid for all $\alpha$ in the domain of $\Psi$, namely $\alpha>-\lambda_{P}$.

In order to identify which $Q$-functions have a given $\mu$-invariant measure, it will be necessary to explain how $\mu$-invariant and $\mu$-subinvariant measures can be identified using resolvents. If $P$ is an arbitrary $Q$-function with resolvent $\Psi$ and $m=\left(m_{j}, j \in S\right)$ is a $\mu$-subinvariant measure for $P$, where of necessity $\mu \leqslant \lambda_{P}$ (see Lemma 4.1 of [22]), then, since the integral (6) converges for all $\alpha>-\lambda_{P}$, we have that, for all $j$ in $S$ and $\alpha>0$,

$$
\sum_{i \in S} m_{i} \alpha \psi_{i j}(\alpha-\mu) \leqslant m_{j}
$$

with equality for all $j$ and $\alpha$ if $m$ is $\mu$-invariant for $P$. One may, therefore, refer to $m$ as being $\mu$-subinvariant for $\Psi$ if (16) is satisfied and $\mu$-invariant if it is satisfied with equality. The following result establishes a characterisation of $\mu$-invariance and $\mu$-subinvariance for $P$ in terms of $\Psi$.

Lemma 1. Let $m$ be a measure on $S$ and let $P$ be a standard transition function with resolvent $\Psi$. Then, if $m$ is $\mu$-subinvariant for $P$, it is $\mu$-subinvariant for $\Psi$ and strictly $\mu$-invariant for $\Psi$ if it is $\mu$-invariant for $P$. Conversely, if $\mu \leqslant \lambda_{P}$ and $m$ is $\mu$-subinvariant for $\Psi$, then $m$ is $\mu$-subinvariant for $P$ and strictly $\mu$-invariant for $P$ if it is $\mu$-invariant for $\Psi$.

Proof: We need only show that the $\mu$-subinvariance and, then, $\mu$-invariance of $m$ for $\Psi$ implies that the same is true for $P$. So, suppose that $m$ is $\mu$-subinvariant for $\Psi$, where $\mu \leqslant \lambda_{P}$, and define $\Psi^{*}$ by

$$
\psi_{i j}^{*}(\alpha)=\frac{m_{j} \psi_{j i}(\alpha-\mu)}{m_{i}}, \quad i, j \in S, \alpha>0
$$

Then, it is easy to verify that $\Psi^{*}$ satisfies (7)-(10). Condition (10) is immediate. Conditions (7) and (9) hold because it is clear, from the definition of $\Psi$, that $\Psi$ satisfies (7) for all $\alpha>-\lambda_{P}$ and (9) for all $\alpha, \beta>-\lambda_{P}$. And, Condition (8) is satisfied by virtue of (16). Thus, $\Psi^{*}$ is the resolvent of a unique (standard) transition function, $P^{*}$. Now define $\widetilde{P}(\cdot)=\left(\tilde{p}_{i j}(\cdot), i, j \in S\right)$ by

$$
\tilde{p}_{i j}(t)=e^{\mu t} \frac{m_{j} p_{j i}(t)}{m_{i}}, \quad i, j \in S, t \geqslant 0,
$$


and $\tilde{\Psi}(\cdot)=\left(\tilde{\psi}_{i j}(\cdot), i, j \in S\right)$ by

$$
\tilde{\psi}_{i j}(\alpha)=\int_{0}^{\infty} e^{-\alpha t} \tilde{p}_{i j}(t) d t, \quad i, j \in S, \alpha>0 .
$$

Then, for all $i, j \in S$ and $\alpha>0$,

$$
\begin{aligned}
\tilde{\psi}_{i j}(\alpha) & =\int_{0}^{\infty} e^{-(\alpha-\mu) t} \frac{m_{j} p_{j i}(t)}{m_{i}} d t \\
& =\frac{m_{j} \psi_{j i}(\alpha-\mu)}{m_{i}} \\
& =\psi_{i j}^{*}(\alpha) .
\end{aligned}
$$

Thus $\tilde{\Psi}=\Psi^{*}$, and hence, from Reuter's characterisation, $\widetilde{P}=P^{*}$. Since $P^{*}$ satisfies (3), it follows immediately that $m$ is $\mu$-subinvariant for $P$. Further, we see that $m$ is $\mu$-invariant for $P$ if and only if $P^{*}$ is honest. Thus, if $m$ is $\mu$-invariant for $\Psi$, then $\Psi^{*}$ is honest and so the ensuing honesty of $P^{*}$ implies that $m$ is $\mu$-invariant for $P$.

I shall now suppose that $m$ is a prescribed $\mu$-subinvariant measure for $Q$ and then, using Theorem 1, I shall determine for which $Q$-functions, $P$, other than $F$, can $m$ be a $\mu$-invariant measure; notice that if $m$ is $\mu$-invariant for a $Q$-function, then, by the minimality of $F$, it is $\mu$-subinvariant for $F$ and so, by Proposition 1 of [21], it must be $\mu$-subinvariant for $Q$.

THEOREM 2. Let $Q$ be a stable, conservative, single-exit $q$-matrix over an irreducible state-space, $S$, and suppose that $m$ is a $\mu$-subinvariant measure on $S$ for $Q$. Let $\Phi(\cdot)=\left(\phi_{i j}(\cdot), i, j \in S\right)$ be the resolvent of $F$, the minimal $Q$-function. Define $z(\cdot)=\left(z_{i}(\cdot), i \in S\right)$ by

$$
z_{i}(\alpha)=1-\sum_{j \in S} \alpha \phi_{i j}(\alpha), \quad i \in S, \alpha>-\lambda_{F},
$$

and $d(\cdot)=\left(d_{i}(\cdot), i \in S\right)$ by

$$
d_{i}(\alpha)=m_{i}-\sum_{j \in S} m_{j}(\alpha+\mu) \phi_{j i}(\alpha), \quad i \in S, \alpha>-\mu .
$$

Then there exists a $Q$-function, $P$, for which $m$ is $\mu$-invariant if and only if $d=0$ or, otherwise,

$$
\left(\frac{\alpha}{\alpha+\mu}\right) \sum_{i \in S} d_{i}(\alpha) \leqslant \sum_{i \in S} m_{i} z_{i}(\alpha)<\infty
$$


for all $\alpha>-\mu$. When such a $Q$-function exists it is unique and its resolvent, $\Psi(\cdot)=$ $\left(\psi_{i j}(\cdot), i, j \in S\right)$, is given by

$$
\psi_{i j}(\alpha)=\phi_{i j}(\alpha)+\frac{z_{i}(\alpha) d_{j}(\alpha)}{(\alpha+\mu) \sum_{k \in S} m_{k} z_{k}(\alpha)}, \quad i, j \in S .
$$

It is then the unique honest $Q$-function for which $m$ is $\mu$-invariant if and only if

$$
\left(\frac{\alpha}{\alpha+\mu}\right) \sum_{i \in S} d_{i}(\alpha)=\sum_{i \in S} m_{i} z_{i}(\alpha)
$$

for all $\alpha>-\mu$.

REMARK: The condition $d=0$ is essentially known (see [21] and [13]). If $d \neq 0$ then $d(\cdot)=\left(d_{i}(\cdot), i \in S\right)$ gives the deficit in the $\mu$-subinvariance of $m$ for $\Phi$; notice that if $m$ is $\mu$-invariant for $P$ then, by the minimality of $F$, it must be strictly $\mu$ subinvariant for $F$ and, hence, for $\Phi$, and so $d_{i}(\alpha)>0$ for all $i$ and for all $\alpha>-\mu$.

Proof: First observe that, since $m$ is $\mu$-subinvariant for $Q$, Proposition 2 of [21] implies that $m$ is $\mu$-subinvariant for $F$ and so, by Lemma 1 , it is $\mu$-subinvariant for $\Phi$. Thus $d_{i}(\alpha) \geqslant 0$ for all $i \in S$ and $\alpha>-\mu$. Further, since $m$ is $\mu$-subinvariant for $F$, it follows, from Lemma 4.1 of [22], that $\mu \leqslant \lambda_{F}$.

Let $P$ be an arbitrary $Q$-function with resolvent $\Psi$ specified by Theorem 1 . I shall show that the stated condition is necessary for $m$ to be $\mu$-invariant for $P$. So, suppose that $m$ is $\mu$-invariant for $P$ and, hence, for $\Psi$. If $P=F$, then $m$ is $\mu$-invariant for $\Phi$ and it follows immediately that $d=0$. To deal with the case $P \neq F$, first observe that, by the minimality of $F, m$ cannot be $\mu$-invariant for $F$, and so $d_{i}(\alpha)>0$ for all $i$ and $\alpha$. Too, neither $z$ nor $y$ in (12) is identically zero. If $\alpha>0$ then $\alpha-\mu$ lies in the domain of $\Psi$ since, of necessity, $\mu \leqslant \lambda_{P}$. Thus, on substituting $\alpha-\mu$ for $\alpha$ in (12), multiplying by $\alpha m_{i}$ and, then, summing over $i \in S$, we find that

$$
\sum_{i \in S} m_{i} z_{i}(\alpha)<\infty
$$

for all $\alpha>-\mu$, and, further, that

$$
m_{j}=\sum_{i \in S} m_{i} \alpha \phi_{i j}(\alpha-\mu)+\alpha y_{j}(\alpha-\mu) \sum_{i \in S} m_{i} z_{i}(\alpha-\mu)
$$

for all $\alpha>0$. Hence, in view of (13), we require

$$
\frac{(\alpha+\mu) \eta_{j}(\alpha)}{c+\sum_{k \in S} \alpha \eta_{k}(\alpha)}=\frac{d_{j}(\alpha)}{\sum_{i \in S} m_{i} z_{i}(\alpha)}
$$


for all $\alpha>-\mu$. But, since (14) must hold and because we require $c \geqslant 0$, we must have that

$$
\left(\frac{\alpha}{\alpha+\mu}\right) \sum_{i \in S} d_{i}(\alpha) \leqslant \sum_{i \in S} m_{i} z_{i}(\alpha)
$$

for all $\alpha>-\mu$. Thus, (18) is necessary for $m$ to be $\mu$-invariant for $P$ when $P \neq F$.

Conversely, if $d=0$ then $m$ is $\mu$-invariant for $\Phi$ and so, on recalling that $\mu \leqslant \lambda_{F}$, it follows, from Lemma 1 , that $m$ is $\mu$-invariant for $F$; by the minimality of $F, m$ is $\mu$-invariant for no other $Q$-function. If $d \neq 0$ and (18) holds, then, in order to construct the resolvent of a $Q$-function, $P$, for which $m$ is $\mu$-invariant, define $\eta$ by

$$
\eta_{j}(\alpha)=d_{j}(\alpha), \quad j \in S, \alpha>-\mu \text {. }
$$

Clearly (14) is satisfied and, using the resolvent equation for $\Phi$, it is easy to show that (15) holds. Thus, in order to specify a $Q$-resolvent, it remains only to determine a value of $c$ so as to be consistent with (13). This can be done as follows:

Using the resolvent equation for $\Phi$, it is easy to show that $z$ and $d$ satisfy

$$
z_{i}(\alpha)-z_{i}(\beta)+(\alpha-\beta) \sum_{k \in S} \phi_{i k}(\alpha) z_{k}(\beta)=0
$$

for all $\alpha, \beta>-\lambda_{F}$, and

$$
d_{i}(\alpha)-d_{i}(\beta)+(\alpha-\beta) \sum_{k \in S} d_{k}(\alpha) \phi_{k i}(\beta)=0,
$$

for all $\alpha, \beta>-\mu$. On multiplying the first equation by $m_{i}$ and summing over $i$, we find that

$$
(\alpha+\mu) \sum_{i \in S} m_{i} z_{i}(\alpha)-(\beta+\mu) \sum_{i \in S} m_{i} z_{i}(\beta)=(\alpha-\beta) \sum_{i \in S} d_{i}(\alpha) z_{i}(\beta),
$$

for all $\alpha, \beta>-\mu$. Similarly, summing the second equation over $i$ gives

$$
\alpha \sum_{i \in S} d_{i}(\alpha)-\beta \sum_{i \in S} d_{i}(\beta)=(\alpha-\beta) \sum_{i \in S} d_{i}(\alpha) z_{i}(\beta), \quad \alpha, \beta>-\mu
$$

Thus

$$
(\alpha+\mu) \sum_{i \in S} m_{i} z_{i}(\alpha)-\alpha \sum_{i \in S} d_{i}(\alpha)=(\beta+\mu) \sum_{i \in S} m_{i} z_{i}(\beta)-\beta \sum_{i \in S} d_{i}(\beta), \quad \alpha, \beta>-\mu
$$

and so, if the sums converge, then

$$
(\alpha+\mu) \sum_{i \in S} m_{i} z_{i}(\alpha)-\alpha \sum_{i \in S} d_{i}(\alpha)
$$


is the same for all $\alpha>-\mu$. Thus, since (18) is satisfied, we may set $c$ equal to this quantity and then arrive at the specification (19) of a $Q$-resolvent which is valid for all $\alpha>-\mu$. Multiplying (19) by $(\alpha+\mu) m_{i}$ and summing over $i$ shows that $m$ is $\mu$ invariant for $\Psi$. Now, as the domain of $\Psi$ must contain $(\mu, \infty)$ it follows that $\mu \leqslant \lambda_{P}$, where $\lambda_{P}$ is the decay parameter of $P$, and, hence, that $m$ is $\mu$-invariant for $P$. To see that $P$ is the unique $Q$-function for which $m$ is $\mu$-invariant, observe that if $m$ is to be $\mu$-invariant for an arbitrary $Q$-resolvent, $\widehat{\Psi}$, then, in view of (21), we must have (in an obvious notation) that $\widehat{\eta}=K d$ for some positive scalar function $K$. Now, on substituting $\widehat{\eta}$ into (21) we find (again, using an obvious notation) that $K(\alpha) c=\widehat{c}$ for all $\alpha$. Thus $K$ is constant, and, moreover,

$$
\frac{\widehat{\eta}_{j}(\alpha)}{\widehat{c}+\sum_{k \in S} \alpha \widehat{\eta}_{k}(\alpha)}=\frac{d_{j}(\alpha)}{(\alpha+\mu) \sum_{i \in S} m_{i} z_{i}(\alpha)}
$$

Thus, $\Psi$ is the unique $Q$-resolvent for which $m$ is $\mu$-invariant.

Finally, the condition for the existence of a unique honest $Q$-function follows on observing that $\Psi$ is honest if and only if $c=0$.

I shall complete this section by looking at the important special case where $m$ can be normalised to produce a probability distribution over $S$; under certain conditions $m$ can then be interpreted as a quasistationary distribution (see, for example, [23]).

Corollary 1. A sufficient condition for the existence of a unique $Q$-function for which $m$ is $\mu$-invariant is that

$$
\sum_{i \in S} m_{i}<\infty
$$

It is honest if and only if $\mu=0$.

ProOF: First observe that, since $z_{i}(\alpha) \leqslant 1$, we have that

$$
\sum_{i \in S} m_{i} z_{i}(\alpha)<\infty
$$

for all $\alpha>-\mu$. On summing over $i$ in (17) we find that (18) is satisfied and, in particular, that

$$
\left(\frac{\alpha}{\alpha+\mu}\right) \sum_{i \in S} d_{i}(\alpha)=\sum_{i \in S} m_{i} z_{i}(\alpha)-\left(\frac{\mu}{\alpha+\mu}\right) \sum_{i \in S} m_{i}
$$

for all $\alpha>-\mu$. Finally, (20) holds if and only if $\mu=0$. 


\section{REFERENCES}

[1] K.L. Chung, 'On the boundary theory for Markov chains', Acta Math. 110 (1963), 19-77.

[2] K.L. Chung, 'On the boundary theory for Markov chains II', Acta Math. 115 (1967), 111-163.

[3] Dobrušin, 'Some classes of homogeneous denumerable Markov processes', Teor. Veroyatnost. i Primenen 2 (1957), 377-380.

[4] J.L. Doob, 'Markov chains - denumerable case', Trans. Amer. Math. Soc. 58 (1945), 455-473.

[5] W. Feller," 'Boundaries induced by stochastic matrices', Trans. Amer. Math. Soc. 83 (1956), 19-54.

[6] W. Feller, 'On boundaries and lateral conditions for the Kolmogoroff differential equations', Ann. Math. 65 (1957), 527-570.

[7] D.G. Kendall, 'Some further pathological examples in the theory of denumerable Markov processes', Quart. J. Math. Oxford 7 (1956), 39-56.

[8] D.G. Kendall and G.E.H. Reuter, 'Some pathological Markov processes with a denumerable infinity of states and the associated semigroups of operators on $l$ ', Proc. Internat. Congr. Math. Amsterdam 1954 III (1954), 377-415.

[9] J.F.C. Kingman, 'The exponential decay of Markov transition probabilities', Proc. London Math. Soc. 13 (1963), 337-358.

[10] W. Ledermann and G.E.H. Reuter, 'Spectral theory for the differential equations of simple birth and death processes', Philos. Trans. Roy. Soc. London 246 (1954), 321-369.

[11] J. Neveu, 'Lattice methods and submarkovian processes', Proc. Fourth Berkley Symp. of Math. Statist. and Probab. II (1961), 347-391.

[12] J. Neveu, 'Sur les états d'entrée et les états fictifs d'un processus de Markov', Ann. Inst. H. Poincaré Pobab. Statist. 17 (1962), 324-337.

[13] P.K. Pollett, 'On the equivalence of $\mu$-invariant measures for the minimal process and its q-matrix', Stochastic Process. Appl. 22 (1986), 203-221.

[14] P.K. Pollett, 'Reversibility, invariance and $\mu$-invariance', Adv. in Appl. Probab. 20 (1988), 600-621.

[15] P.K. Pollett, 'Invariant measures for $Q$-processes when $Q$ is not regular', Adv. in Appl. Probab. 23 (1991) (to appear).

[16] G.E.H. Reuter, 'Denumerable Markov processes and the associated contraction semigroups on l', Acta Math. 97 (1957), 1-46.

[17] G.E.H. Reuter, 'Denumerable Markov processes (II)', J. London Math. Soc. 34 (1959), 81-91.

[18] G.E.H. Reuter, 'Denumerable Markov processes (III)', J. London Math. Soc. 37 (1962), 63-73.

[19] G.E.H. Reuter, 'Note on resolvents of denumerable submarkovian processes', $Z$. Wahrscheinlichkeitstheorie 9 (1967), 16-19.

[20] G.E.H. Reuter and W. Ledermann, 'On the differential equations for the transition probabilities of Markov processes with enumerably many states', Proc. Camb. Phi. Soc. 49 
(1953), 274-262.

[21] R.L. Tweedie, 'Some ergodic properties of the Feller minimal process', Quart. J. Math. Oxford 25 (1974), 485-495.

[22] D. Vere-Jones, 'Ergodic properties on non-negative matrices I', Pacific J. Math. 22 (1967), 361-386.

[23] D. Vere-Jones, 'Some limit theorems for evanescent processes', Austral. J. Statist. 11 (1969), 67-78.

[24] D. Williams, 'The process extended to the boundary', Z. Wahrscheinlichkeitstheorie 2 (1963), 332-339.

[25] D. Williams, 'On the construction problem for Markov chains', Z. Wahrscheinlichkeitstheorie 3 (1964), 227-246.

[26] Yang Xiangqum, 'Construction of Q-processes satisfying Kolmogoroff's backward equations in single exit case of forward equations in single entrance case', Kexue Tongbao 26 (1981), 390-394.

Department of Mathematics

The University of Queensland

Queensland 4072

Australia 